\title{
Changes in respiration during emotional stress
}

\author{
Alberto Hernando ${ }^{1,2}$, Jesús Lázaro ${ }^{1,2}$, Adriana Arza ${ }^{2,3}$, Jorge Mario Garzón ${ }^{2,3}$, Eduardo Gil ${ }^{1,2}$, \\ Pablo Laguna ${ }^{1,2}$, Jordi Aguiló ${ }^{2,3}$, Raquel Bailón ${ }^{1,2}$ \\ ${ }^{1}$ BSICoS Group, Aragón Institute of Engineering Research (I3A), IIS Aragón, University of \\ Zaragoza, Zaragoza, Spain, \\ ${ }^{2}$ CIBER de Bioingeniería, Biomateriales y Nanomedicina (CIBER-BBN), Zaragoza, Spain \\ ${ }^{3}$ Microelectronics and Electronic Systems Department. Autonomous University of Barcelona, \\ Bellaterra, Spain
}

\begin{abstract}
In this work, we analyze changes in respiration during emotional stress induced by a modification of the Trier Social Stress Test. The following stages in the test were analyzed: the pre-relaxing stage, the story telling stage, the anticipation of stress and the video exposition stage. Respiration signal is recorded during the whole test using a thoracic band. Power spectral density (PSD) of respiration is computed in running windows using a modification of Welch periodogram in which sufficiently peaked spectra are averaged. Then, respiratory frequency $\left(F_{R}\right)$ is estimated from the "peaked-conditioned" averaged spectra. Results show that respiratory rate is significantly $(p<0.05$ according to the Friedman test) higher, while a measure of spectral peakness and the percentage of PSD used to compute $\left(F_{R}\right)$ is lower during stress stages than during relax. These results suggest that the respirationrelated parameters have potential discrimination power for stress level assessment.
\end{abstract}

\section{Introduction}

Stress is defined as a physiological response to a threatening situation and is considered a survival mechanism. However, when it is prolonged or repetitive, it has been associated with psychic and somatic diseases among others. Chronic stress has been related with higher risk of suffering a cardiovascular diseases [1].

Unfortunately, the identification of stress is non trivial. There are not objective methods for stress measurement, the symptoms vary among subjects and the personal perception of each one counts too. There is the need to investigate methods towards an objective measure of stress.

Respiration is known to be related to stress. An increase in respiratory frequency $\left(F_{R}\right)$ when stress level is higher has been reported during mental load and sustained atten- tion [2]. Relationship between stress and respiration is so close that in some articles the voluntary control of respiration is studied to reduce the negative effects of stress [3]. In this work we analyze changes in respiration during emotional stress induced by a modification of the Trier Social Stress Test (TSST) [4] in young healthy subjects.

\section{Materials and methods}

\subsection{Data collection}

A data base of 25 volunteers ( 9 men and 16 women) with an age of $21.56 \pm 2.71$ is used. These registers were recorded in the Autonomous University of Barcelona [5].

Emotional stress is induced following a modification of the Trier Social Stress Test [4] approved by the Ethics Committee, including the following stages:

- Pre-relaxing stage (PR): 10 minutes of relaxing audition.

- Story telling stage (ST): 3 stories are told to the subject with a great amount of details. The subject is requested to remember as much details as possible, creating a stressful situation.

- Memory task (MT): the subject has to tell back the 3 stories with all the details that he/she remembers in $30 \mathrm{sec}-$ onds for each story.

- Stress anticipation (SA): subject is waiting during 10 minutes for the evaluation of the memory test done before. - Video display (VD): the projection of a video with the subject performance in the memory test is showed. Prior to each subject's performance review, the video of an actor remembering all the details is displayed.

- Arithmetic Task (AT): the subject has to perform successive subtractions of 13 , starting from the number 1022. In case of a calculation error, the countdown is restarted from 1022. The subject has 5 minutes to accomplish the task.

Respiration signal is recorded and sampled at $250 \mathrm{~Hz}$ using Medicom system, ABP-10 module (Medicom MTD Ltd, Russia). 
PR and SA have longer duration that the other stages. Only the 6 central minutes of PR and SA are analyzed in this work.

\subsection{Respiratory frequency estimation}

Respiration signal is filtered with a pass-band filter (cutoff frequencies of 0.03 and $0.9 \mathrm{~Hz}$ ), downsampled to $4 \mathrm{~Hz}$ and independently analyzed in the 6 time stages.

In order to find the respiratory rate, the algorithm described in [7] is applied. The method consists in the estimation of the respiratory frequency $\left(F_{R}\right)$ from "peakedconditioned" averaged spectra.

First, spectra are estimated using Welch periodogram in running 40 seconds-window with 8 seconds-overlap, denoted $S_{k}(f)$. A measure of peakness is obtained from $S_{k}(f)$ as:

$$
P_{k}=\frac{\int_{F_{R}(k-1)-\delta}^{F_{R}(k-1)+\delta} S_{k}(f) d f}{\int_{0.08}^{0.8} S_{k}(f) d f} * 100
$$

where $F_{R}(k-1)$ denotes the estimated respiratory rate at the $(k-1)^{t h}$ instant; $\delta$ value is experimentally fixed as $0.1 \mathrm{~Hz}$. Then, a peaked-conditioned average spectra $\left(\overline{S_{k}(f)}\right)$ is obtained averaging those $S_{k}(f)$ which are sufficiently peaked, defined in this work as $P_{k}>65 \%$. Figure 1 displays two spectra, one with $P_{k}>65 \%$ entering the averaging, and another one with $P_{k}<65 \%$, not used in the peaked-conditioned average.
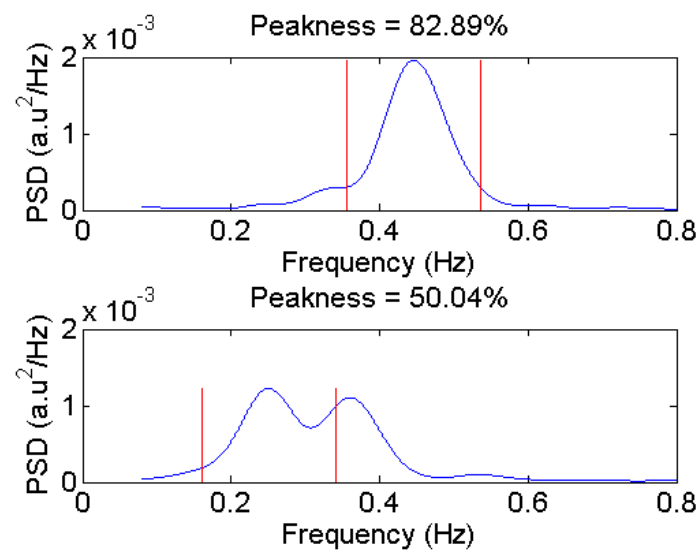

Figure 1. Differences between computed and non computed spectra to estimate respiratory frequency according to its peakness.

Finally, respiratory frequency is estimated from the peaked-conditioned averaged $\overline{S_{k}(f)}$ in a frequency band dependent on previous respiratory frequency estimated, as described in [7].

Not only it is interesting to study respiratory frequency changes related to stress, but other parameters related to respiratory regularity and variability, such as the peakness measure and the percentage of spectra which take part in the peaked -conditioned averaging.

This method successfully estimates respiratory frequency in 4 out of 6 stages of the stress test, namely PR, ST, SA and VE. In MT and AT speaking modifies the respiratory pattern [8] and spectra are not sufficiently peaked, except from some intervals in a few subjects ( 2 in $\mathrm{M}$ and 6 in AT).

\subsection{Statistical analysis}

In order to study a single value per stage in each subject, the median of all the intra-subject-measurements in each stage is computed. The statistical test is applied over: respiratory rate median $F_{R}^{m}$; peakness measure median $P_{k}^{m}$; and percentage of spectra used to compute the peakedconditioned averaging. Towards the comparative of the different moments of the same subject a Friedman test is used, since the studied parameters have non-normal distributions. P-values lower than 0.05 are considered significant.

\section{Results}

Figure 2 shows differences in respiratory frequency of one subject between PR and ST, which results to be the one that has the highest frequency rate (note that MS and AT are few and very short registers). Upper images displays one-minute respiratory wave in both stages; medium images, all the periodograms; lower images, only the averaged periodograms where respiratory rate is going to be estimated.

General results are shown in Table 1, computing the median and the median absolute deviation among the 25 subjects. It is shown that during stress stages respiratory rate is higher than during relax ( $* p<0.05$ according to the Friedman test). The peakness and the percentage of spectra used to compute respiratory frequency are analyzed to show the signal stability. These values are higher in relax than in the other stages.

Table 1. Median \pm mad of $F_{R}^{m}, P_{k}^{m}$ and $\% S_{k}(f)$ from all subjects in the different parts of the test.

\begin{tabular}{|c|c|c|c|c|}
\hline Stage & PR & ST & SA & VE \\
\hline \multirow{2}{*}{$R_{F}^{m}$} & 0.25 & 0.34 & 0.3 & 0.34 \\
& \pm 0.07 & $\pm 0.05^{*}$ & $\pm 0.04^{*}$ & $\pm 0.04^{*}$ \\
\hline \multirow{2}{*}{$P_{k}^{m}$} & 78.44 & 72.82 & 72.37 & 74.19 \\
& \pm 5.02 & $\pm 8.57^{*}$ & $\pm 8.04^{*}$ & $\pm 8.21^{*}$ \\
\hline \multirow{2}{*}{$\% S_{k}(f)$} & 87.8 & 58.64 & 76.41 & 72.5 \\
& \pm 12.82 & $\pm 12.5^{*}$ & $\pm 12.17^{*}$ & $\pm 10.73^{*}$ \\
\hline
\end{tabular}



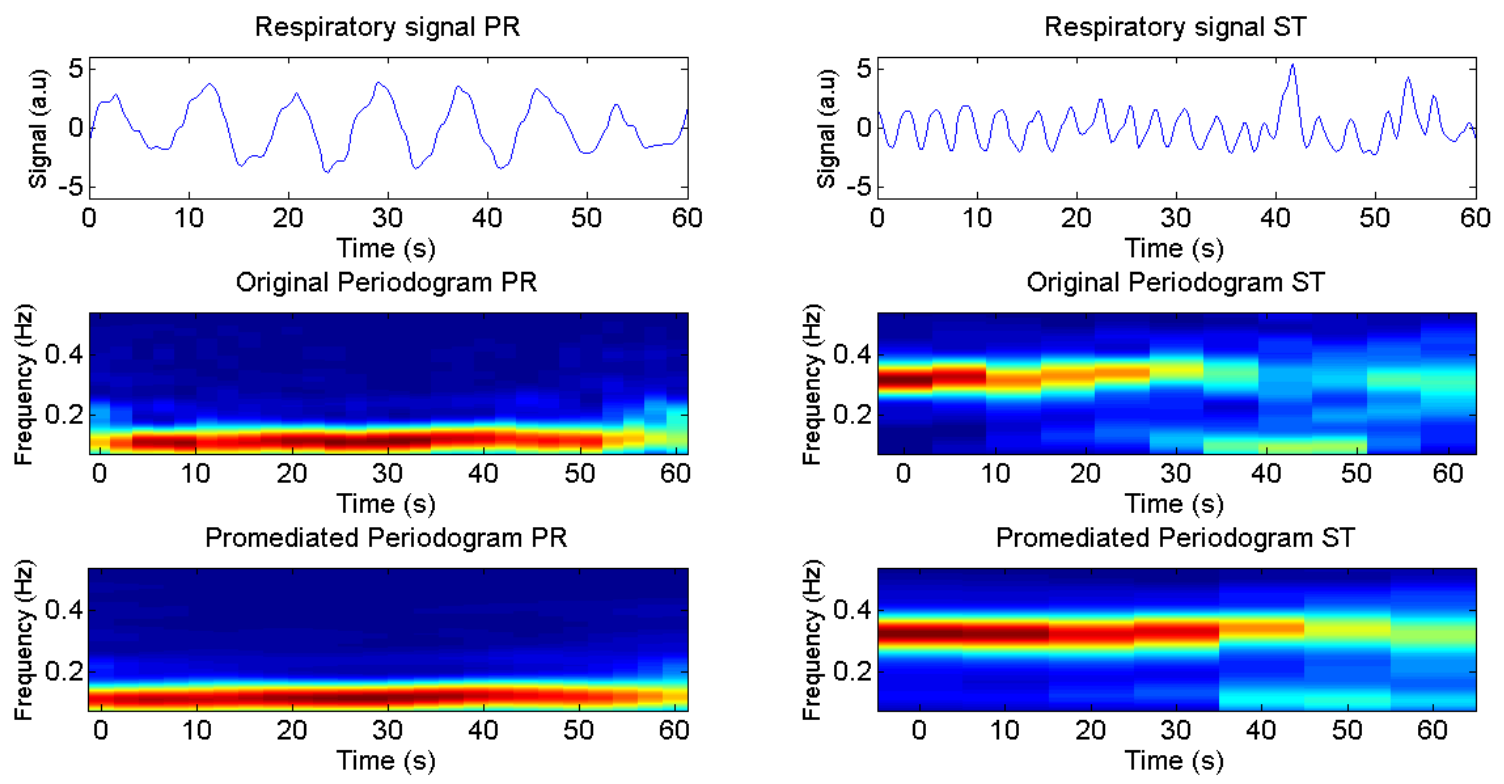

Figure 2. Respiratory signal and periodograms in pre-relaxing stage and story telling.

In the two stages when the subject is speaking, with few subjects computed, median and mad results shows a similar trend. Higher values of frequency rate than in relax: in MT $F_{R}^{m}=0.4 \pm 0.04$ and in $\mathrm{AT} F_{R}^{m}=0.34 \pm 0.07$. Lower values in peakness and percentage of spectra used than in PR, in MT $P_{k}^{m}=70.5 \pm 13.52$ and $\% S_{k}(f)=$ $34.2 \pm 20.35$; in AT $P_{k}^{m}=45.91 \pm 3.76$ and $\% S_{k}(f)=$ $44.52 \pm 20.84$.

\section{Discussion}

Based on the results of this study, respiration changed during all the stages of the stress test with respect to the pre-relaxing stage, in agreement with results reported in [2], where the baseline register presented a lower respiratory rate than in attention or mental arithmetic task.

Spectral peakness and the percentage of spectra accepted to compute the respiratory frequency are studied in this work as measures of respiration stability (variability). The more stable respiration is, the more peaked the spectra are and a higher number of them are included in the average. Results show that both are higher in PR than in the other 3 stages, suggesting more stable respiration during relax than during the stress stages of the test. The less peaked spectra are found in VE, while the fewest spectra are used in ST. In [2] respiratory variation measured as the variation of a breath component over a sampling period of 15 minutes (just the opposite of our stability) is computed as a coefficient that increased with mental task comparing to relax situation.
Not only respiratory information is useful in stress assessment, but it is also important in the analysis and interpretation of heart rate variability (HRV) during stress. In [5] classical spectral HRV index were analyzed in this database. In 7 out of the 25 subjects, respiratory frequency resulted to be below $0.2 \mathrm{~Hz}$ during PR so that power reflecting respiratory sinus arrhythmia may leak in the low frequency (LF) band, leading to an overestimation of sympathetic activity and an underestimation of parasympathetic activity. Thus, knowledge of respiratory frequency could aid in the interpretation of HRV during stress [6].

On the other hand, in MT and in AT respiratory frequency is higher than the upper limit of the classic HF band. This may refer that classic HF band is not a measure of vagal activity in these intervals. Unfortunately, few subjects are analyzed in MT and AT because the speaking makes harder to registry respiratory rate. Therefore, the potential use of other tools to analyze these stages is open.

\section{Conclusion}

In this work, respiratory parameters such as respiratory rate or stability are analyzed and compared during a modification of the Trier Social Stress Test.. Respiratory rate is higher during stress phases than during relax and respiratory stability (studied as its spectral peakness and percentage of spectra used to computed a peaked-conditioned spectra) is lower. This suggests that these parameters are potentially useful in stress level assessment. 


\section{Acknowledgements}

This work is supported by Universidad de Zaragoza under fellowship UZ2014-TEC-01 and PIFUZ-2011-TRCA-003, by Ministerio de Economía y Competitividad (MINECO), FEDER; under projects FIS-PI12/00514 and TIN2014-53567-R, by CIBER in Bioengineering, Biomaterials \& Nanomedicne through Instituto de Salud Carlos III, and by Grupo Consolidado BSICoS from DGA (Aragón) and European Social Fund (EU). The computation was performed by the ICTS "NANBIOSIS', by the High Performance Computing Unit of the CIBER in Bioengineering, Biomaterials \& Nanomedicne (CIBER-BBN) at the University of Zaragoza.

\section{References}

[1] Chandola T, Britton A, Brunner E, Hemingway H, Malik M, Kumari M, Badrick E, Kivimaki M, Marmot M "Work stress and coronary heart disease: what are the mechanisms?" European Heart Journal 29, 5, 640-648, 2008.

[2] Vlemincx E., Taelman J., De Peuter S., Van Diest I., Van den Bergh O. "Sigh rate and respiratory variability during mental load and sustained attention". Psychophysiology 48, vol. 1, pp.117-120, 2011.

[3] Brown R. P., Gerbarg P. L. "Sudarshan Kriya yogic breathing in the treatment of stress, anxiety, and depression: part I-neurophysiologic model." Journal of Alternative and Complementary Medicine 11, vol. 1, pp. 189-201, 2005.
[4] Hellhammer J., Schubert M.,"The physiological response to Trier Social Stress Test relates to subjective measures of stress during but not before or after the test", Psychoneuroendocrinology, vol. 37, no. 1, pp. 119-124, 2012.

[5] Arza A., Garzón J. M., Hernando A., Aguiló J., Bailón R., "Towards an Objective Measurement of Emotional Stress: Preliminary Analysis Based on Heart Rate Variability", IEEE Engineering in Medicine and biology Society, 2015.

[6] R. Bailón, P. Laguna, L. Mainardi and L. Srnmo. "Analysis of Heart Rate Variability Using Time-Varying Frequency Bands Based on Respiratory Frequency". 29th Annual International Conference of the IEEE Engineering in Medicine and Biology Society (EMBC07), 2007, Lyon, CD. pp. 6674-6677.

[7] Lázaro J., Alcaine A., Romero D., Gil E., Laguna P., Pueyo E. Bailón R., "Electrocardiogram Derived Respiratory Rate from QRS Slopes and R-Wave Angle", Annals of Biomedical Engineering, Vol. 40, No. 10, pp. 2072-2083, 2014.

[8] Kumar S., Al'Absi M., Beck J. G., Ertin E., Scott M. S., "Behavioral monitoring and assessment via mobile sensing technologies” In: Marsch L. A., Lord S. E., Dallery J. ”Behavioral Healthcare and Technology". New York: Oxford University Press, 2015:621-4.

Address for correspondence:

Name Alberto Hernando Sanz

Postal address c/ Mariano Esquillor s.n, 50018, Zaragoza, Spain

E-mail address (optional)610631@unizar.es 